\title{
Open Innovation in der Politik: Internetgestützte Zusammenarbeit in politischen Parteien am Beispiel der ÖVP in Österreich
}

\author{
Lisa Schmidthuber (D) - Maximilian Rapp • Niclas Kröger • \\ Dennis Hilgers (iD
}

Eingegangen: 24. August 2018 / Angenommen: 13. Dezember 2018 / Online publiziert: 28. Dezember 2018

(C) Der/die Autor(en) 2018

Zusammenfassung Dieser Artikel liefert Belege dafür, wie eine politische Partei digitale Kommunikationstechnologie nutzt, um ein neues Parteiprogramm gemeinsam mit den Bürgern zu entwickeln. Anhand einer Case Study Analyse wird gezeigt, wie politische Parteien vom digitalen Wandel profitieren und Online Plattformen gezielt für eine bessere Kommunikation mit Bürgern einsetzen können. Eine quantitative Befragung unter jenen, die mit der Partei digital zusammenarbeiten, zeigt, dass sich eine homogene Gruppe von Bürgern an der Initiative beteiligt. Die Teilnehmer wollen die zukünftige Entwicklung des Landes mitbestimmen und weisen ein hohes $\mathrm{Maß}$ an politischem Interesse auf. Die Analyse der Umfragedaten weist darauf hin, dass die Plattform-User die Initiative positiv in Hinblick auf Informationsverbreitung und Diskussion unter den Parteimitgliedern bewerten. Basierend auf der qualitativen und quantitativen Analyse des Projektes werden schließlich praxisnahe Implikationen abgeleitet. Die Handlungsempfehlungen sollen Entscheidungsträger in Parteien dabei unterstützen, einen Mehrwert aus einer digitalen Zusammenarbeit mit Bürgern $\mathrm{zu}$ generieren.

\footnotetext{
L. Schmidthuber $(\bowtie) \cdot$ D. Hilgers

Johannes Kepler Universität Linz, Altenbergerstraße 69, 4040 Linz, Österreich

E-Mail: Lisa.schmidthuber@jku.at

D. Hilgers

E-Mail: dennis.hilgers@jku.at

M. Rapp

Skoltech Institute of Science and Technology, Moscow, Russland

E-Mail: Maximilian.rapp@de.ey.com
}

Ernst \& Young, Arnulfstr. 59, 80636 München, Deutschland

N. Kröger

HYVE - the innovation company, Schellingstraße 45, 80799 München, Deutschland

E-Mail: niclas.kroeger@hyve.net 
Schlüsselwörter Open Government · eGovernment · Open Innovation · Bürgerbeteiligung $\cdot$ Demokratie $\cdot$ ePartizipation

\title{
Open Innovation in Politics: Analyzing Internet-Based Collaboration in an Austrian Political Party
}

\begin{abstract}
Digital platforms are leveraged to exchange with the external environment, benefit from knowledge of online communities, and lastly to co-create value. Similar to companies that invite customers to share their ideas for product development online, open innovation platforms are used by political parties to exchange with their party members. This article provides empirical evidence on how a political party uses communication technology to co-create a new party program with citizens. A case study analysis shows how political parties can benefit from digital transformation and use online platforms for stimulating communication with citizens. A quantitative survey among platform users indicates that a homogeneous group of citizens participated in the inititative who is politically interested and wants to participate in the future development of the country. Based on the qualititative and quantitative analysis of the initiative, practical implications were derived. These recommendations should help decision-makers to generate added value from collaboration with citizens.
\end{abstract}

Keywords Open Government · e-government · Open innovation · Citizen participation $\cdot$ Democracy $\cdot$ e-participation

\section{Einführung}

Im Laufe der letzten Jahre lässt sich verstärkt ein offener Ruf nach mehr Beteiligung und das Verlangen nach digitalen Mitgestaltungsräumen beobachten. Die durch „Open Innovation“ beeinflusste Diskussion, flächendeckend Meinungsbilder durch das Internet abzufragen, hat an Bedeutung gewonnen, als Barack Obama sein großes Engagement „Open White House“ nach seiner ersten Wahl zum US-Präsidenten startete (Collm et al. 2013). Durch die organische und kulturelle Latenz waren Piratenpartei, Online-Bürgerinitiativen und „Liquid Democracy“ ein paar Jahre später in Deutschland Agenda-Setter für Bürgerbeteiligungsthemen auf großer (Bundestag) sowie kleiner (Kommunen) politischer Bühne. Aber auch Herausforderungen gehen mit dem Streben nach mehr Online-Bürgerpartizipation einher: fehlende Transparenz nach Open Government Initiativen, mangelnde Anteilnahme durch zu schwache Kommunikation, mangelnde Qualität durch nicht authentische Kampagnen sind dabei nur einige Gründe für die mangelnde Begeisterung bei Amtsträgern und Bürgern. In Kontrast dazu lösen Unternehmen und Organisationen aus der Privatwirtschaft immer komplexere Themenstellungen mit Kunden sowie Markenfans und bauen bedeutende Kundenkanäle auf, um kollaborativ und kundenzentriert den digitalen Wandel zu meistern (Rapp et al. 2015). 
Dieser erfolgreiche (Online-)Austausch und die daraus resultierende Markentreue sind nur begrenzt in der politischen Landschaft zu finden. Open Government Abstimmungen, digitale Demokratisierungsbewegungen oder politische Gestaltungsfreiheit für aktive Bürger sind somit immer ein Stück Utopie geblieben. Gerade die Parteien hinken den großen Ansprüchen der vor Jahren veröffentlichten lokalen und nationalen Agenden weit hinterher. Als Konsequenz verlagern sich die Diskussionen und Meinungsbilder in dezentrale Blogs, Communities und v. a. soziale Netzwerke, wo sie unkontrolliert und somit auch unkommentiert bleiben. Als Ergebnis bleiben frustrierte Bürger und weitestgehend unqualifizierte Inhalte, die in einer Abwärtsspirale wiederum in größerer Politikverdrossenheit münden.

Nichtsdestotrotz können immer wieder auch erfolgreiche Initiativen zum Thema Bürgerbeteiligung entstehen. Dieser Artikel stellt ein Beispiel von Open Innovation in der Politik vor: Die Initiative „Evolution Volkspartei“ mit der digitalen „Ideenwand"-Plattform der konservativen ÖVP (Österreichische Volkspartei). Ziel des Artikels ist es, Implikationen für die Praxis zu definieren, die als Grundlage für weitere Initiativen aus dem öffentlichen Bereich genutzt werden können, um Fehler zu vermeiden und noch größeren qualitativen Output zu erzeugen. Die Erkenntnisse einer Case Study Analyse und die Ableitungen aus einer quantitativen Studie sollen diesen Artikel zu einer Hilfestellung für Praktiker im Beteiligungsumfeld machen. Folglich werden wir 1.) den Case der ÖVP näher darstellen, 2.) unsere Ergebnisse zu der hierzu durchgeführten quantitativen Studie näher untersuchen sowie 3.) Erfolgsfaktoren und Management-Implikationen für zukünftige und praxisbezogene Open Government bzw. Bürgerpartizipationsprojekte ableiten.

\section{Open Innovation in der Politik}

Crowdsourcing, Online Communities und interaktive Wertschöpfung spiegeln die Ideen von „Open Innovation“ wider (Chesbrough et al. 2006). Demnach können Wissensströme zwischen einer Organisation und ihren externen Partnern bzw. Anspruchsgruppen das Innovationspotential eines Unternehmens bzw. einer Organisation verbessern. Von „Open Innovation“ wird dann gesprochen, wenn Externe wie Kunden oder Produktnutzer dem Unternehmen ihr Lösungs- und Bedürfniswissen zur Verfügung stellen und damit dem Unternehmen helfen, den Innovationsprozess effektiver zu gestalten (Thomke 2003; Piller und Reichwald 2009). Mit Hilfe des externen Wissens können bedarfsgerechte Produkte und Dienstleistungen entwickelt werden, die mit Unternehmenserfolg und Wettbewerbsvorteilen einhergehen. Die Einbeziehung von Externen in den Produktentwicklungsprozess wird dabei durch den Einsatz von digitaler Kommunikationstechnologie erleichtert. Beispielsweise werden digitale Plattformen, mobile Applikationen oder soziale Netzwerke genutzt, um Kommunikation zu ermöglichen und Wissensströme zu organisieren.

Der Ansatz von offenen Organisationsgrenzen wird auch in den öffentlichen Sektor übertragen (,Open Government“) und öffentliche Institutionen sammeln bereits erste Erfahrungen mit kollaborativen Entscheidungsprozessen (Hilgers 2012; Schmidthuber und Hilgers 2018). Im Rahmen von Bürgerhaushalten zum Beispiel werden Bürger eingeladen, Ideen für die Verwendung eines Teilbudgets online vor- 
zubringen. Online-Ideenwettbewerbe werden veranstaltet, um Bürger in die Stadtoder Gebäudeplanung einzubinden. Infrastrukturmängel sollen mit Hilfe von mobilen Applikationen der Stadtverwaltung mitgeteilt werden, um gemeinschaftlich Probleme zu beseitigen.

Der Open Innovation Ansatz kann jedoch nicht nur der öffentlichen Verwaltung, sondern auch anderen Akteuren des politisch-administrativen Systems einen Mehrwert bieten. So dient die Öffnung für externes Wissen auch als Strategie für politische Parteien, um die Distanz zu den Wählern zu überwinden. Politische Parteien können auf externes Wissen (z.B. von Parteimitgliedern) in Bezug auf ihre politischen Visionen und Ideen zurückgreifen. Sie haben Zugang zu einem großen Pool an Erfahrungen, Meinungen und Ideen von Personen, die durch ihre Mitgliedschaft bereits Interesse an der Parteipolitik bekundet haben. Politische Innovation umfasst beispielsweise die Entwicklung neuer politischer Visionen, Ziele, Strategien und Programme. Letzteres enthält die grundlegenden Forderungen, Ziele und Werte einer politischen Partei. Insbesondere führen Wahlniederlagen dazu, dass politische Parteien ihre Identität durch Neuformulierung des Parteiprogramms überdenken (z. B. Pautz 2009). Dieser Artikel skizziert im Folgenden, wie die ÖVP mit Hilfe von Open Innovation Instrumenten ein neues Parteiprogramm gemeinsam mit Bürgern entwickelt hat.

\section{Daten und Methoden}

Um den ÖVP-Case in seiner gesamten Komplexität zu untersuchen und zu verstehen, werden qualitative mit quantitativen Forschungsansätzen kombiniert und somit ein „Mixed-Methods“-Ansatz gewählt (Johnson und Onwuegbuzie 2004). Zur qualitativen Auswertung brachte sich das Forscherteam selbst aktiv in den gesamten Prozess des Bürgerdialogs ein und konnte somit ein tieferes Verständnis für die Thematik und die Wirkzusammenhänge entwickeln (Baskerville und Wood-Harper 1996). Hierbei wurde ein explorativer Ansatz gewählt. Zudem wurde eine Umfrage unter den Plattform-Usern durchgeführt, um zu untersuchen, wer sich an dem Projekt beteiligt, aus welchen Gründen teilgenommen wird und wie die Initiative bewertet wird. Nach einem Pretest wurde allen registrierten Usern eine Einladung zur Teilnahme an der Umfrage per Mail gesendet. Insgesamt wurden 432 Fragebögen vollständig ausgefüllt und für die Analyse herangezogen.

\section{Ergebnisse}

\subsection{Case Study: In sechs Stufen zum neuen Parteiprogramm der ÖVP}

Die ÖVP entschied sich im Jahr 2014 dazu, ein neues Parteiprogramm zu verfassen, da das alte Programm vor zwanzig Jahren entworfen wurde und sich somit unzureichend mit bedeutsamen Themen der Gegenwart befasste. Beispielsweise waren Themen wie die Digitalisierung, Globalisierung oder EU-Entwicklung nicht Teil des damaligen Parteiprogramms. Nachdem andere Parteien wie die CSU (Christ- 
lich-Soziale Union) in Bayern bereits große Erfolge damit verzeichnet hatten, ihr Parteiprogramm gemeinsam mit den Bürgern zu kreieren (Rapp et al. 2015), beschloss auch die ÖVP, die schriftlichen Grundsätze ihrer Partei gemeinsam mit den Bürgern zu entwickeln. Im Gegensatz zur Schwesterpartei CSU, die zuvor lediglich Parteimitglieder in den Prozess eingebunden hatte, wählte die ÖVP einen in Teilen komplett offenen Ansatz, in welchen sich alle interessierten Bürger aktiv einbringen konnten.

Insgesamt lässt sich der „Evolution Volkspartei“ - so der Name der Initiative genannte Prozess in sechs Schritte unterteilen (vgl. Abb. 1):

1. Themenidentifikation: Auf Veranstaltungen mit Bürgern sammelten die Schirmherren der Initiative verschiedene Themengebiete, die von großem Interesse in der Bevölkerung waren.

2. Themenauswahl: Aus diesen Erkenntnissen wurden parteiintern die wichtigsten ausgewählt und zu elf Diskussionsbereichen zusammengefasst. Diese umfassten alle relevanten sozialen, wirtschaftlichen und politischen Themenfelder.

3. Ideengenerierung und Diskussion: Auf einer Online-Plattform, der sog. Ideenwand (vgl. Abb. 2), konnten sich Bürger registrieren, ihre Ideen für Inhalte des Parteiprogramms einreichen und die Beiträge anderer Einreicher bewerten und diskutieren. Zudem bestand die Möglichkeit, Offline-Events mit Hilfe der Plattform zu initiieren und schließlich zu koordinieren.

4. Auswahl der Fragestellungen: Basierend auf den Diskussionen auf der Plattform wurden insgesamt 39 Leitfragen zur Abstimmung ausgewählt. Die Kondensation der Ideen zu diesen Leitfragen fand innerhalb der Partei statt.

5. Abstimmung: In diesem Schritt konnten Parteimitglieder der ÖVP (zu diesem Zeitpunkt ca. 650.000) abstimmen, ob die in den Leitfragen enthaltenen Aussagen ins Parteiprogramm aufgenommen werden sollten. 12.835 Parteimitglieder beteiligten sich an der Abstimmung.

6. Parteiprogramm: Auf Grundlage der Inhalte der Onlineplattform und den Ergebnissen der Abstimmung wurde ein neues Parteiprogramm vom Kern der Partei geschrieben.

Der Fokus dieses Artikels liegt im Besonderen auf den Teilschritten 3 und 5, welche durch Online-Komponenten eine Einbindung der Bevölkerung ermöglichten. Die Ideensammlung und Diskussion auf der „Ideenwand“ Plattform (3. Teilschritt) sind hierbei als Kernbestandteil der Bürgerbeteiligung anzusehen.

Die „Ideenwand“ Plattform, in deren Konzeption sich die Forschergruppe aktiv eingebracht hat, bot der Bevölkerung einen nutzerfreundlichen Zugang zur Teilnahme an der Diskussion. Nach einer einfachen Registrierung, bei der Name, Postleitzahl, E-Mail-Adresse und optional die Parteinummer angegeben werden konnten, waren die Bürger in der Lage, sich aktiv in die Diskussion des Parteiprogramms einzubringen. Sie konnten eigene Beiträge schreiben, Beiträge von anderen Teilnehmern kommentieren und bewerten (,gefällt mir“/,gefällt mir nicht“). Zudem konnten sie Interesse an Offline-Veranstaltungen bekunden oder ihre eigenen erstellen, die in einem Kalender mit Kartenfunktion dargestellt wurden. 


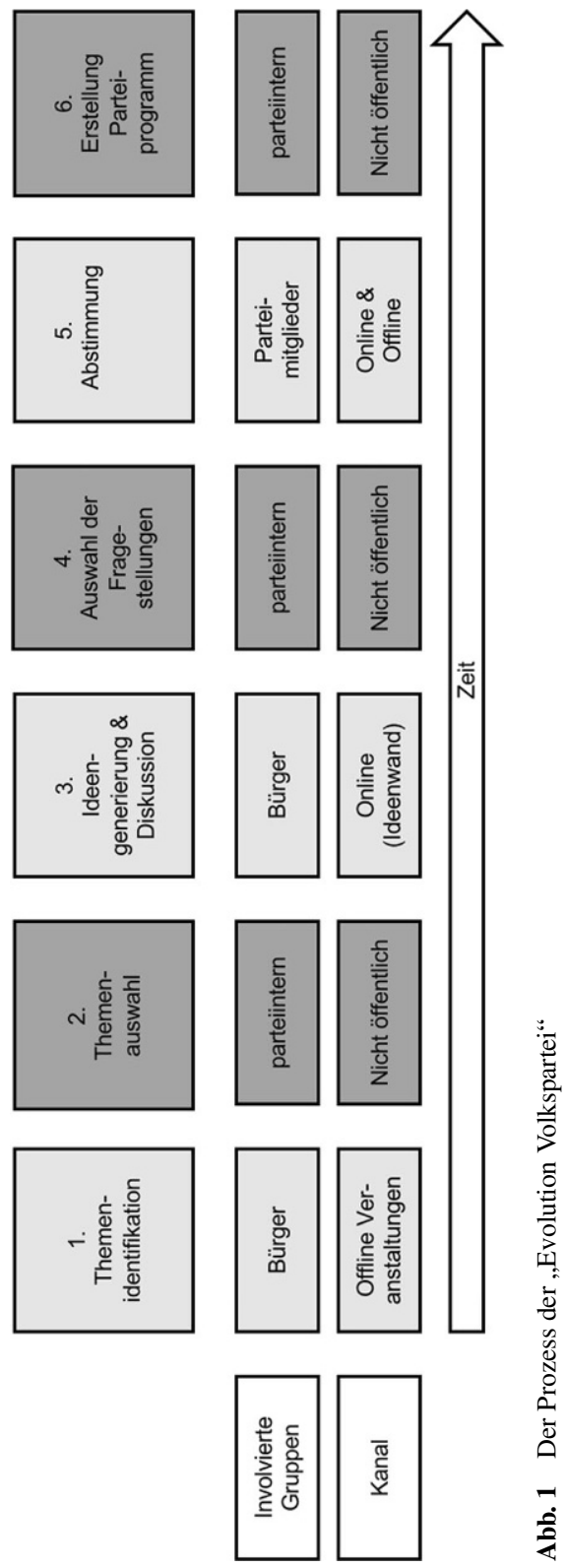

Obwohl die Partei in den meisten Phasen der Initiative die Bevölkerung aktiv in die Entwicklung einband, war die Partizipation nicht im gesamten Prozess möglich. Die Teilnehmer konnten Ideen einreichen und kommentieren. Parteimitgliedern war es möglich, sowohl online als auch offline, über die Leitfragen abzustimmen. Die wichtigen Entscheidungen, wie die Auswahl der Themen, das Zusammenfassen zu Leitfragen und die Erstellung des finalen Parteiprogramms wurden jedoch innerhalb 
Abb. 2 Screenshot der Ideenwand Plattform zur Online-Bürgerbeteiligung

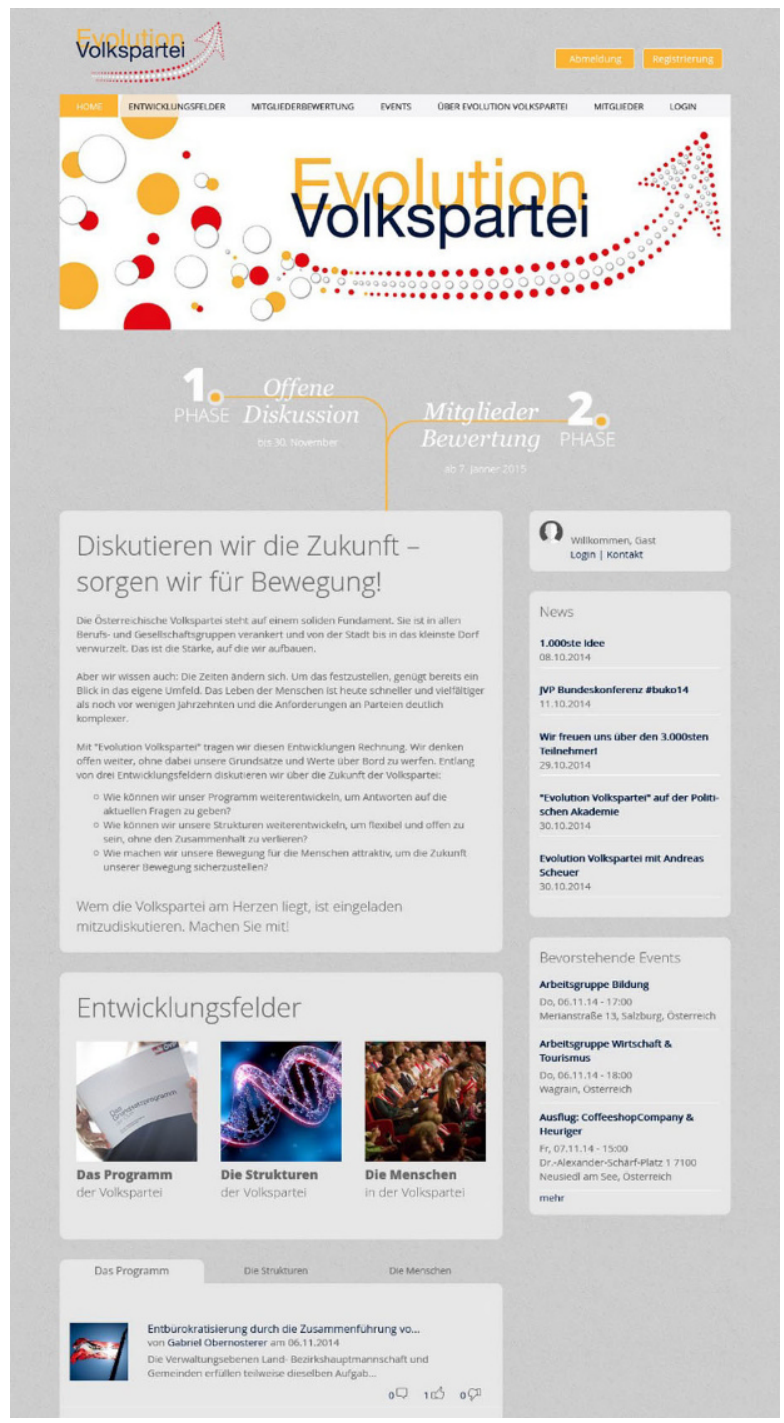

der Partei getroffen. Die Erstellung des neuen Parteiprogramms lässt sich mit einem klassischen Stage-Gate-Prozess vergleichen, in dem die Partei als Gatekeeper in den Teilschritten 2, 4 und 6 agiert.

Die Möglichkeit für jeden Bürger in den Prozess der Erstellung des Parteiprogramms, der traditionell komplett in der Hand der Partei liegt, aktiv einzugreifen, ist ein Fortschritt in Richtung einer Öffnung der Partei. Dies zeigt sich auch in der Quantität der Teilnehmer und Beiträge. 5337 Bürger erstellten sich ein Profil auf der Plattform und schrieben 2949 Beiträge, die sie 6537-mal kommentierten. Zudem gaben die Teilnehmer ihre Meinung zu den Beiträgen in knapp 12.000 ,gefällt mir“ bzw. ,gefällt mir nicht“ ab. Auch wurde die Möglichkeit, begleitende Offline-Events 
Abb. 3 Beispielhafte Leitfrage

\section{Pflege}

Soll sich die ÖVP dafür aussprechen, dass die höheren Kosten für die Pflege durch eine eigene zusätzliche Pflegeversicherung aller Erwerbstätigen abgedeckt werden?

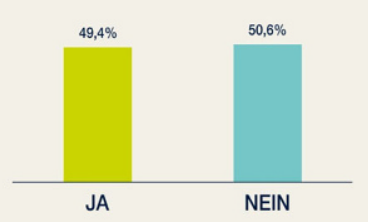

zu besuchen bzw. selbst zu organisieren und über die Plattform bekannt zu machen, von den Bürgern stark genutzt. Insgesamt wurden 157 Veranstaltungen erstellt und durchgeführt. Ein wichtiger Faktor in der Erreichung dieser eindrucksvollen Kennzahlen stellten die sogenannten Botschafter dar. Über 40 Parteifunktionäre machten es sich zur besonderen Aufgabe, die Initiative im ganzen Land bekannt zu machen. Einer dieser Botschafter war Sebastian Kurz, der damalige Außenminister, der 2017 zum österreichischen Bundeskanzler gewählt wurde. Eine solch hohe Besetzung der Botschafterposten signalisierte auch die Relevanz der „Evolution Volkspartei“ gegenüber der Bevölkerung.

Schließlich wurde versucht, die Qualität des Prozesses durch ein hohes Maß an partizipativer Entscheidungsfindung sicherzustellen. So wurden die 39 durch die Partei identifizierten Leitfragen fast ausnahmslos von den Parteimitgliedern übernommen. Die Themen dieser Fragen, die aus den Plattforminhalten abgeleitet wurden, umfassten hierbei eine Vielzahl relevanter Themengebiete, wie Zugangsbeschränkungen für Universitäten, Familienbild, Gesundheit, Entbürokratisierung, Europa und Energie. Lediglich eine Frage zur Pflege wurde knapp abgelehnt (siehe Abb. 3). Die hohe Qualität der Einreichungen, die als Grundlage für die Leitfragen diente, erklärt sich möglicherweise durch die Sicherstellung eines konstruktiven Diskurses auf der Plattform. Hierzu wurden Community Manager speziell ausgebildet, die die Diskussion leiten und für eine positive Stimmung in der Community sorgen konnten. Um ein tieferes Verständnis für das Verhalten der Teilnehmer zu entwickeln, brachte sich auch die Forschergruppe aktiv in das Community Management ein.

\subsection{Umfrageergebnisse}

\subsubsection{Merkmale der Plattorm-User}

Nach dem Ende der „Evolution Volkspartei“ wurden die Plattform-User eingeladen, an einer Umfrage zur Evaluierung der Initiative teilzunehmen. 432 der registrierten User nutzten diese Möglichkeit und füllten den Fragebogen aus. Tab. 1 gibt einen 
Tab. 1 Soziodemografische Merkmale der Befragten

\begin{tabular}{llllll}
\hline Variable & $N$ & $\%$ & Variable & $N$ & $\%$ \\
\hline Gender & 428 & 100 & Bildungsniveau & 426 & 100 \\
Männlich & 367 & 85,75 & Gering & 81 & 19,01 \\
Weiblich & 61 & 14,25 & Mittel & 109 & 25,59 \\
Geburtsjahr & 413 & 100 & Hoch & 236 & 55,40 \\
$1935-1956$ & 104 & 25,18 & Wahlpräferenz & 407 & 100 \\
$1957-1976$ & 224 & 54,24 & ÖVP & 325 & 79,85 \\
$1977-1997$ & 85 & 20,58 & Andere Partei & 82 & 20,15 \\
\hline
\end{tabular}

Überblick über die demografischen Charakteristika der Stichprobe. Über $85 \%$ der Befragten sind männlich und mehr als die Hälfte der Teilnehmer sind zwischen 40 und 60 Jahre alt. Rund $25 \%$ der Befragten sind älter als 60 Jahre. Des Weiteren weist die deskriptive Analyse auf ein hohes Bildungsniveau der Teilnehmer hin. Mehr als $50 \%$ der Befragten haben einen Hochschulabschluss. Schließlich gaben etwa $80 \%$ der Befragten an, dass sie bei der nächsten Wahl die ÖVP wählen würden. Die verbleibenden $20 \%$ nahmen an der Entscheidungsfindung teil, würden sich bei der nächsten Wahl aber für eine andere Partei entscheiden.

\subsubsection{Gründe der Teilnahme}

Die Gründe für die Teilnahme an Open Innovation können sehr vielseitig sein. Abb. 4 zeigt als Hauptmotiv, den Wunsch der Befragten, sich an der Entwicklung des österreichischen Gemeinwesens zu beteiligen. Ein Großteil der Befragten nennt weiter die Parteimitgliedschaft als Grund, an dem kollaborativen Innovationsprozess teilzunehmen. Interesse an der Parteipolitik und die Möglichkeit, direkt mit der Partei und Gleichgesinnten zu kommunizieren, stellen zusätzliche Motivationsfaktoren der Teilnahme an „Evolution Volkspartei“ dar. Während der Wunsch nach Austausch und partizipativer Entscheidungsfindung die Hauptmotive der Befragten zu sein scheinen, spielen intrinsische Motivation wie Spaß und Freude an der Nutzung der Plattform oder Erfahrung mit ähnlichen Plattformen eine untergeordnete Rolle.

\subsubsection{Bewertung des Open Innovation Prozesses}

Abb. 5 gibt einen Überblick über die Beurteilung der Initiative von Seiten der Teilnehmer. Insgesamt zeigt sich, dass die Befragten die Initiative in Hinblick auf Informationsverbreitung und Diskussion unter den Parteimitgliedern positiv bewerten. Die Initiative schaffte eine Plattform zum Austausch der Parteimitglieder untereinander und führte nach Meinung der Befragten zu einer verbesserten Entscheidungsfindung. Die gemeinsame Entscheidungsfindung geht jedoch auf Kosten der Effizienz der Erstellung eines neuen Parteiprogramms. Neben einer intensiven Diskussion unter den Teilnehmern sollte die Initiative auch mit einer höheren Zufriedenheit mit der Partei und deren Entscheidungen einhergehen. Die Meinung der Befragten dazu ist geteilt. Knapp $40 \%$ der Befragten sind der Meinung, dass der Kollaborationsprozess zu mehr Vertrauen in die Partei und zu einer höheren Zufriedenheit mit 


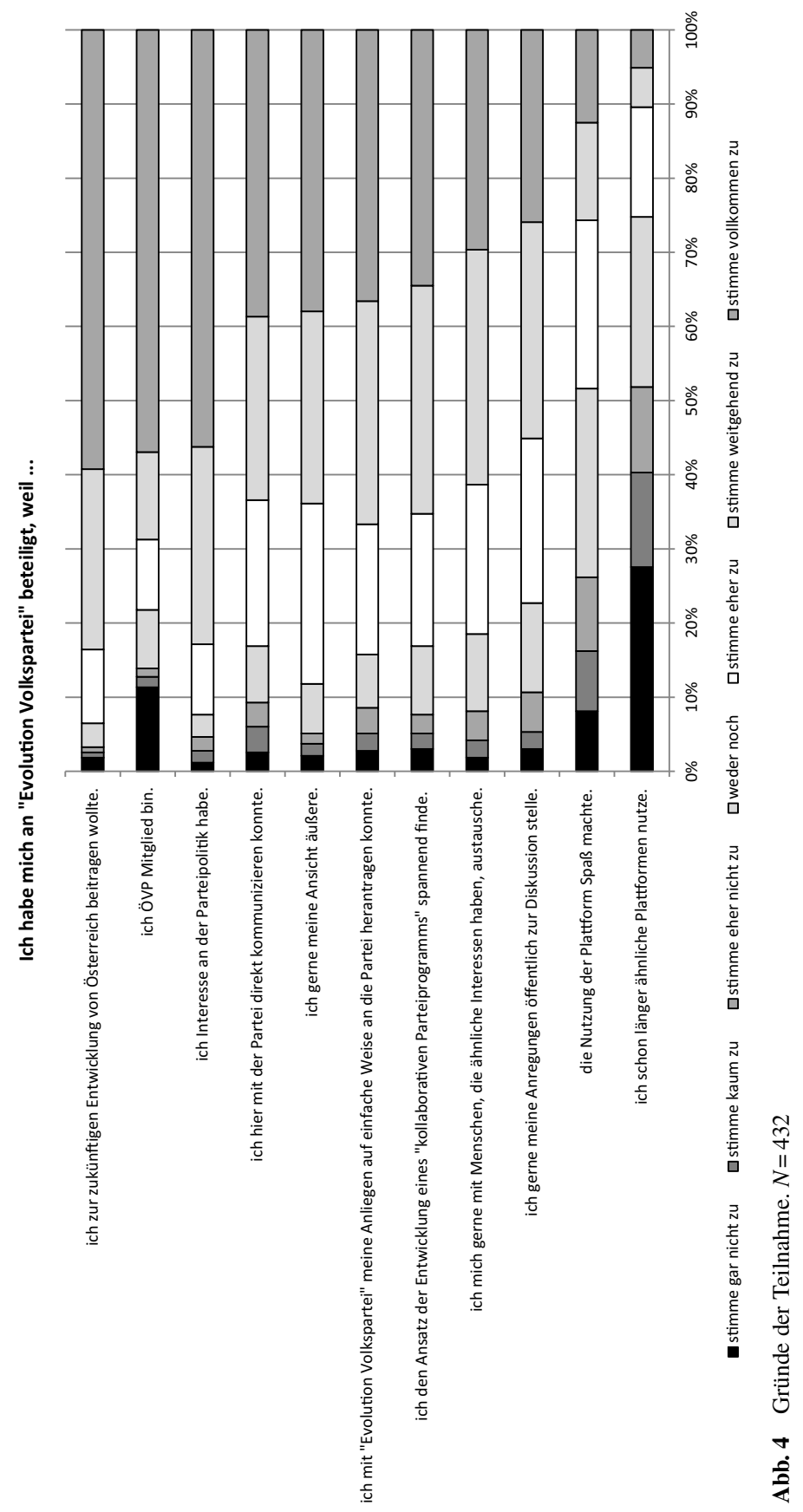




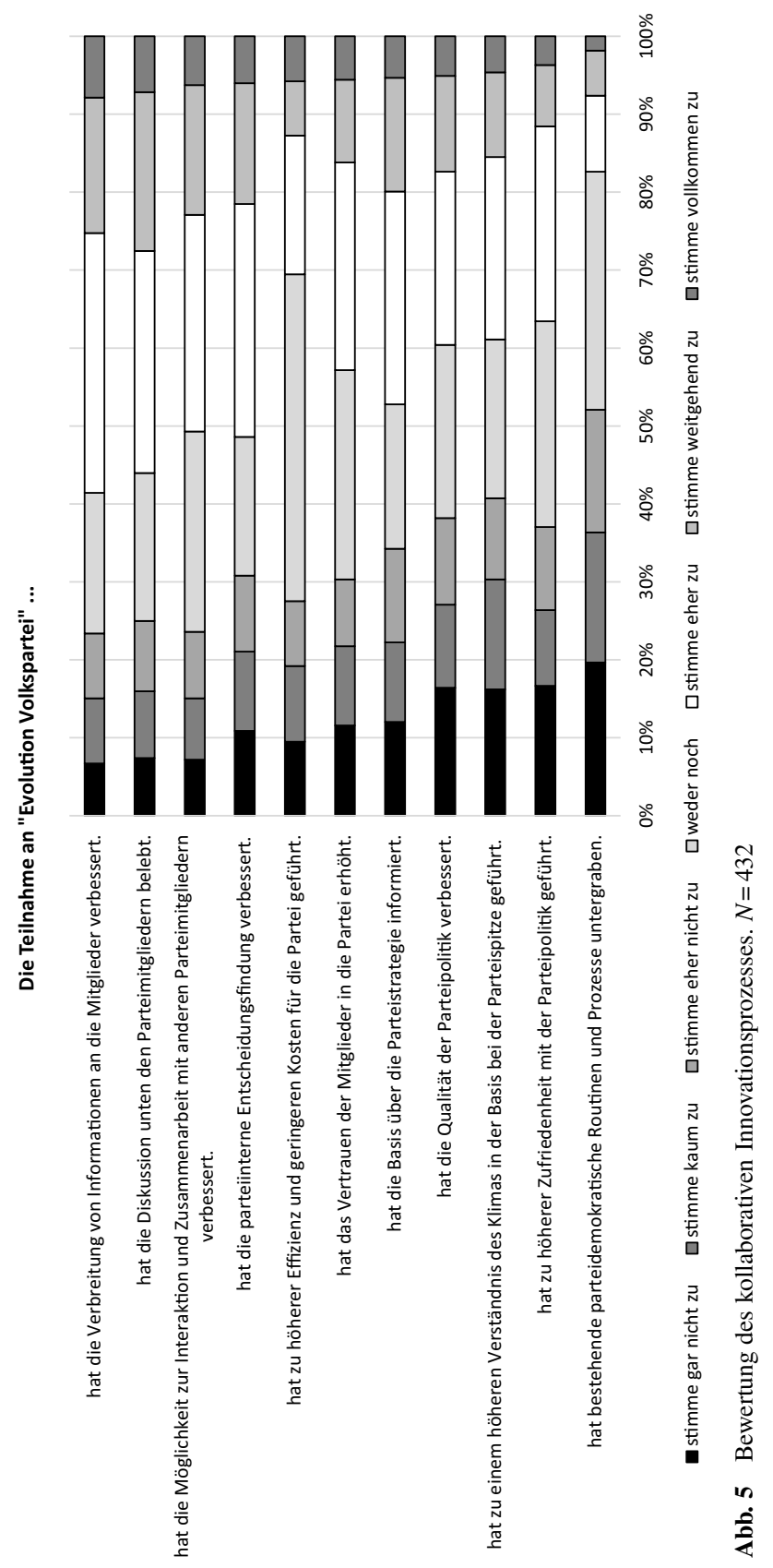


der Parteipolitik geführt hat. Zusammengefasst lässt sich festhalten, dass die Initiative als ein Mittel gesehen wird, die Kommunikation und den Austausch unter den Parteimitgliedern zu beleben, sie jedoch weniger dazu führt, die Zufriedenheit mit der Parteipolitik zu verbessern. Hierfür scheint eine einmalige Initiative nicht ausreichend zu sein.

\section{Diskussion und Implikationen}

\subsection{Diskussion}

Die vorliegende Studie beschäftigt sich zum einen mit der Frage, wie eine politische Partei digitale Kommunikationstechnologien nutzen kann, um Parteimitglieder und Interessierte in die Entwicklung eines neuen Parteiprogramms einzubinden. Um politische Innovation im digitalen Zeitalter besser zu verstehen, wurde in dem vorliegenden Artikel ein österreichisches Beispiel für Open Innovation in der Politik vorgestellt. Bei der Initiative „Evolution Volkspartei“ entschloss sich die mittlerweile amtierende Regierungspartei ÖVP im Jahr 2014, das neue und ,evolutionäre“ Parteiprogramm entgegen der bisherigen Erfahrung nicht unter Ausschluss der Öffentlichkeit durch die Spitzenpolitiker zu schreiben, sondern offen mit externem Wissen zu crowdsourcen. Der Input der Teilnehmer - mit und ohne Parteibuch war dabei quantitativ aber auch qualitativ so bedeutend, dass schlussendlich 39 Leitsätze des neuen Programms der Community entnommen wurden.

Die vorliegende Untersuchung zeigt, wie eine politische Partei der Leitidee von „Open Innovation“ folgt und interessierte Externe einlädt, ihre Ideen für die Formulierung eines neuen Parteiprogramms online zu teilen. Die digitale Plattform dient dem Informationsaustausch unter den Parteimitgliedern, aber auch der Diskussion unter politisch Interessierten. Die Fallstudie zeigt somit, wie ein offener Innovationsansatz in einem politischen Kontext konzipiert und umgesetzt werden kann.

Zum anderen betrachtet die Studie jene Personen, die sich an der Erstellung eines neuen Parteiprogramms beteiligen. Die Ergebnisse der deskriptiven Statistik geben Hinweise darauf, dass eine homogene Gruppe der Online-Community beitritt. Der Großteil der User ist männlich, gut gebildet und zwischen 40 und 60 Jahren alt. Außerdem geht hervor, dass etwa $20 \%$ der Plattform-User Wahlpräferenzen für eine andere Partei als die ÖVP haben. Das bedeutet, dass diese zur Entwicklung einer politischen Agenda beitragen, die sie nicht durch ihre Stimme unterstützen wollen.

Des Weiteren wird gezeigt, dass Personen mit Interesse an „Offline-Politik“ aus unterschiedlichen Gründen einer Online-Community beitreten. Ein Großteil der User hat sich registriert, da sie die Plattform als ein Instrument zur einfacheren Kommunikation zwischen der Partei und ihren Mitgliedern schätzen und zur Gestaltung des neuen Parteiprogramms, als ein wichtiges politisches Instrument in Hinblick auf die Zukunft des Landes, beitragen wollen. Viele User nehmen nicht nur am Projekt teil, weil ihnen die Plattform gut gefällt, sondern auch weil sie sich mit Gleichgesinnten austauschen wollen. 


\subsection{Management Implikationen}

Durch die aktive Begleitung des vollständigen Prozesses der Initiative, durch die daraus gewonnenen Erfahrungen sowie durch den Vergleich mit ähnlichen Projekten aus dem öffentlichen Sektor wollen wir im Folgenden die identifizierten Erfolgsfaktoren für die Durchführung von Open Government Initiativen zusammenfassen:

\section{Parteiinterne Innovationskultur und Top-Down Entscheidungen:}

Analog zu Unternehmen sollte auch innerhalb der politischen Parteien und öffentlichen Organisationen bereits ein gewisses Maß an Innovation und digitalem Wandel stattgefunden haben, um die Herausforderungen einer solchen Initiative zu bewerkstelligen. Die Auswahl der richtigen IT-Plattform, Staffing von Community Managern, kurze Response-Zeiten oder das gemeinsame Innovieren mit dem Bürger sind jedoch oft nicht Teil des klassischen Arbeitsalltags von Führungskräften und Entscheidungsträgern in politischen Parteien, können jedoch das Innovationspotential nachhaltig erhöhen. Darüber hinaus gilt es trotz eines „Bottom-Up Community Gedankens“ innerhalb des Managements klare Top-Down Entscheidungen zu treffen, um eine solche Initiative überhaupt möglich zu machen, innerparteiliche Befürchtungen und Bedenken frühzeitig durch Führung im Keim zu ersticken und agil derartige Herausforderung zu lösen. Denn, sowohl bei anderen Open Government Projekten als auch im wirtschaftlichen Kontext zeigt sich, dass Projekten immer wieder Entscheidungsentschlossenheit und klare Leitlinien fehlen und etwaige Diskussionen $\mathrm{zu}$ einem zeitlichen Verzug führen.

\section{Schnittstelle IT-Abteilung und digitale Plattform:}

Die vorliegende Fallstudie zeigt, dass die gewählte Plattform alleine keinen Erfolg gewährt, Mängel und Bugs beim Bürger jedoch disqualifizieren. Technische Schnittstellen mit weiteren Netzwerken oder Diensten sind ein weiterer Erfolgsfaktor, um schnell eine kritische Masse zu erreichen (beispielsweise Google Maps für Veranstaltungen). Ob die Plattform durch externe Dienstleister oder die interne ITAbteilung bereitgestellt wird, ist sekundär und mehr noch eine Frage der Ressourcen. Besonders wichtig ist jedoch die interne Schnittstelle zwischen IT-Abteilung und der Projektleitung der Initiative. Wird die IT-Abteilung nicht schon ab der IdeationPhase in das Projekt eingebunden, kann 1.) wichtige Zeit durch falsche Anforderungen an die IT-Abteilung verloren gehen, 2.) das kreative Potential der IT-Abteilung zu möglichen Problemfeldern nicht genutzt werden sowie 3.) Ownership bei der ITAbteilung fehlen, wodurch das Interesse der Abteilung und damit auch die Qualität nachhaltig vermindert werden.

\section{Innovationsprozess und Stakeholdermanagement:}

Der Stage-Gate Prozess wird sowohl in der Wirtschaft als auch in der Politik immer stärker durch das Open Innovation Modell verdrängt. Dadurch werden nicht nur externe Ressourcen wie Bürger eingebunden, sondern auch das lineare Entscheidungsmodell innerhalb der Parteien durchbrochen. Dabei braucht es in der Politik weniger unabhängige Organisationformen (Start-Up Umfeld) oder Accelaratoren. Interne Entscheidungen zu wichtigen Zeitpunkten machen durchaus Sinn, um die 
Kontrolle nicht gänzlich an den Bürger abzutreten. Adaptionen im Rahmen des bisherigen Innovationsprozesses (u. a. Scrum, Design Thinking) sind dabei vorteilhaft, um agil mit dem Bürger in offenen oder halboffenen Formaten zusammenzuarbeiten und den teilweise zähen inhaltlichen Entwicklungsprozess maßgeblich zu beschleunigen. Diese Elemente spielten bei der ÖVP - im Gegensatz zu vorherigen Initiativen der Partei - eine bedeutende Rolle und wurden nunmehr langfristig etabliert. Auch die Vielzahl an teils erfolglosen Beispielen aus Deutschland (z. B. Online-Bürgerhaushalte) zeigen, dass Projekte eher als isolierte Initiativen realisiert und eben nicht der Gesamtprozess der Stadt, politischen Partei oder einer anderen Organisation im Rahmen eines Innovationsprogramms grundlegend hinterfragt und überarbeitet wurden. Dabei sollten zudem frühzeitig die richtigen Stakeholder eingebunden werden, da es im Laufe des Projektes durch interne Machtkämpfe (z. B. durch Ansprüche auf den Projekterfolg) zu immer stärkeren Reibungsverlusten kommen kann, die die initiale Zeitschiene massiv beeinflussen. Auch die Auswahl eines - und v.a. des richtigen - Projektleiters, welcher durch Jour Fixe kontinuierlich alle nötigen Stakeholder zur richtigen Zeit einbindet, ist ein wesentlicher Erfolgsfaktor. Diese Form von Projekten sollte nicht als „Nebenjob“ eines Verantwortlichen mitlaufen.

\section{Offene und transparente Kommunikation:}

Die Kommunikation zwischen den durchführenden Organisationen und den Bürgern startet nicht erst mit dem „Go-Live“ der technologischen Beteiligungsplattform, sondern vielmehr bereits zuvor bei den Pressekonferenzen und dem Rekrutieren der Teilnehmer. Da sich die Community eigentlich niemals als Selbstläufer erweist und Mund-zu-Mund Propaganda gerade zu Beginn oft nicht ausreichend ist, bedarf es einer Kommunikationsstrategie - weit vor dem offiziellen Start. Dabei sollten neben Newslettern und E-Mail Verteilern auch innovative Methoden wie bezahlte Werbung oder neue Social Media Formate (z. B. Stories) bedacht werden. Wichtig ist hierbei zudem, dass die Absichten und die Bedeutung der Initiative transparent dargestellt werden. So sollte auch die Nicht-Umsetzung von Ideen offen kommuniziert werden. Wird der Bürger abgeholt, entstehen so auch im Nachgang keine negativen Bumerang-Effekte. Des Weiteren sollten initiale Inhalte auf der Plattform vorab eingestellt werden, um den Eindruck einer lebhaften Community von Beginn an zu suggerieren.

\section{Online und offline Koordination:}

Obgleich die Online-Bürgerbeteiligung naturgemäß die Digitalität impliziert, sollten Offline-Elemente strategisch für Rekrutierung, Motivation und Qualitätsförderung während des Projektes flankierend und gegenseitig befruchtend durchgeführt werden. Dabei spielen gerade Offline-Veranstaltungen eine große Rolle. Hier können z.B. durch Design Thinking Workshops besonders motivierte User eingeladen und im Rahmen von qualitativen Face-to-Face Diskussionen dazu gebracht werden, weiteres Wissen und Erfahrungen zu teilen. Auch das Treffen von Teilnehmern im ,echten“ Leben untereinander, so hat das ÖVP-Projekt gezeigt, ist wichtig, um Themen weiterzuführen und ein zusätzliches Level der Konzepte zu stimulieren. Gerade auch die Vermarktung und die Rekrutierung von Teilnehmern können dabei durch die Mixtur aus innovativen digitalen sowie klassischen Offline-Kanälen wie Plakaten, Flyern und Pressemitteilungen stark profitieren. Online-Beteiligung ist ein 
starkes Tool, Nachhaltigkeit wird jedoch gerade hier noch durch Augenkontakt und den direkten Austausch erzeugt. Die Plattform ist somit immer als Herzstück einer flankierenden Kommunikations- und Integrationsstrategie zu verstehen.

\section{Aktive Community und Bürgerbetreuung:}

Eines der kritischsten Erfolgsprinzipien der Durchführung ist die Betreuung der Teilnehmer, aber auch der geteilten Inhalte auf der Plattform. Während die Auswahl und das Set-Up des technologischen Tools überwiegend Selbstzweck sind, muss die Community durchgehend motiviert, incentiviert und „,bei Laune gehalten“ werden. Dazu ist das Community-Management nicht nur als besseres „Begrüßen und Bedanken“ zu verstehen. Hier liegt die entscheidende Aufgabe, Incentivierungsstrukturen für intrinsisch und extrinsisch motivierte Teilnehmer $\mathrm{zu}$ etablieren sowie die geteilten Inhalte an die verantwortlichen internen Fachstellen der Partei zu verteilen und Feedback abzustimmen. Die inhaltliche Diskussion führt dabei in der Regel zu Überarbeitungen und schließlich zu höherer Qualität der Beiträge.

\section{Authentische Implementierungsbemühungen:}

Ein Grund für die wellenförmige Nachfrage und das Interesse an Online-Bürgerbeteiligung der letzten Jahre ist sicherlich die Vielzahl an Initiativen, die von Beginn an überwiegend aus Wahlkampf- und Marketingzwecken durchgeführt wurden. Interessanterweise steigen die Initiativen vor Wahlen an - ein risikoreiches Unterfangen, das dem Bürger nicht zu entgehen scheint. Im Gegenteil sind diese sehr feinfühlig, investieren sie doch sehr viel Zeit mit ihren Gedanken und Ideen und wollen damit schließlich auch etwas verändern. Doch viele Initiativen starten bereits ohne große Implementierungsabsichten. Daher sollten Initiativen nur als Pilot-Phasen gesehen werden und daraus langfristig Möglichkeiten der Integration entstehen. So lassen sich auch einfache Kampagnen oder Meinungsumfragen gezielt einsetzen, um dauerhafte und somit authentische Beteiligung zu fördern. Partikulare Initiativen sind prinzipiell eher nur dann empfehlenswert, wenn sie eine strategische und einflussreiche Implikation mit sich tragen - wie bei der ÖVP.

\section{Budget und Ressourcen:}

Im Verlauf solch großer Projekte zeigt sich oft, dass Ressourcen in Form von Budget oder Manpower vorab unterschätzt wurden. Um Bürgerbeteiligung erfolgreich und auch langfristig zu betreiben, um ein nachhaltiges Ergebnis zu schaffen, sollten Kostenpunkte wie Community Management, Technologie (egal ob interne Aufwände oder externe Plattformen), Projektmanagement, Kommunikationsmaterialien, mögliche Incentivierungen, Events und Presseveranstaltungen sowie interne Koordinierungsressourcen vorab eingeplant und durchgerechnet werden. Ohne das nötige Budget und die entsprechenden Ressourcen wird es schwierig, die generierten Ideen zu implementieren und im Anschluss an das Projekt dem Bürger die von ihm ko-kreierte Innovationen zur Verfügung zu stellen. 


\section{Fazit}

Der Einsatz von Open Innovation ermöglicht Unternehmen, öffentlichen Organisationen und auch politischen Parteien das Bedürfnis- und Lösungswissen der Kunden, Bürger oder Parteimitglieder zu nutzen, um das organisationale Innovationspotential zu erhöhen und darüber hinaus einen Mehrwert für die Gesellschaft zu generieren. Eine große Herausforderung ist es dabei, moderne Informations- und Kommunikationstechnologie so einzusetzen, dass aus dem Öffnungsprozess eine erfolgreiche Zusammenarbeit zwischen Plattformbetreiber und Beitragenden entsteht.

Diese Arbeit demonstriert am Beispiel der digitalen ,Ideenwand“-Plattform der Österreichischen Volkspartei, wie eine Online-Plattform genutzt werden kann, um ein neues Parteiprogramm gemeinsam zu entwickeln. Der Ansatz der Öffnung der Partei- bzw. Organisationsgrenzen eignet sich allerdings nicht nur zur gemeinsamen Entscheidungsfindung von Parteistrategien. Aus einem einmaligen Projekt mit einem vordefinierten Ablaufdatum kann eine auf Dauer angelegte Maßnahme entstehen, die Wähler abseits der Wahlurne an parteiinternen Entscheidungen teilhaben zu lassen.

Open Innovation in der Politik wirft jedoch noch offene Fragen auf. Eine Herausforderung ist die Datenmenge, die auf Open Innovation Plattformen generiert wird. Zum einen zeichnet sich ein erfolgreiches Open Innovation Projekt durch rege Teilnahme aus, zum anderen steht die Partei nach Einreichschluss vor einer enormen Herausforderung, die Inhalte zu sortieren, nach deren Zweckmäßigkeit zu prüfen und wertvolle Beiträge in das Endprodukt zu integrieren. Dieser Assimilationsprozess muss noch genauer betrachtet werden. Zudem stellt der Umgang mit nichtwertvollen Inhalten eine Herausforderung dar. Was passiert mit Inhalten, die nicht berücksichtigt werden können? Wie reagieren Plattform-User, wenn ihre Beiträge in der finalen Version des Parteiprogrammes nicht $\mathrm{zu}$ finden sind? Des Weiteren zeigt diese Studie abermals, dass sich eine sehr homogene Gruppe an der Initiative beteiligt. Dies hat zur Folge, dass den Stimmen von männlichen, gut gebildeten Personen mittleren Alters mehr Gewicht gegeben wird. Obwohl Open Innovation in der Politik keine Form der direkten Demokratie darstellt, fungiert es als Instrument Meinungsbilder abzufragen. Aufgrund des Participation Gap ist dieses Bild jedoch verzehrt. Neben Faktoren, die Bürger dazu bewegen, mit Parteien auf Plattformen zusammenarbeiten (Schmidthuber et al. 2017), ist somit zukünftig mehr Forschung notwendig, die die Gründe für die Nicht-Teilnahme untersucht und der zentralen Frage nachgeht, ob die Nicht-Teilnahme eine Folge des Nicht-Könnens (z.B. kein Zugang zu Internet, keine ausreichenden Computerkenntnisse) oder des Nicht-Wollens (kein Interesse) darstellt.

Funding Open access funding provided by Johannes Kepler University Linz.

Open Access Dieser Artikel wird unter der Creative Commons Namensnennung 4.0 International Lizenz (http://creativecommons.org/licenses/by/4.0/deed.de) veröffentlicht, welche die Nutzung, Vervielfältigung, Bearbeitung, Verbreitung und Wiedergabe in jeglichem Medium und Format erlaubt, sofern Sie den/die ursprünglichen Autor(en) und die Quelle ordnungsgemäß nennen, einen Link zur Creative Commons Lizenz beifügen und angeben, ob Änderungen vorgenommen wurden. 


\section{Literatur}

Baskerville RL, Wood-Harper AT (1996) A critical perspective on action research as a method for information systems research. J Inf Technol 11(3):235-246

Chesbrough H, Vanhaverbeke W, West J (Hrsg) (2006) Open innovation: researching a new paradigm. Oxford University Press, Oxford

Collm A, Rapp M, Koch G (2013) Erfolgskriterien für die Open Government-Umsetzung: Eine vergleichende Analyse von Parteien und Gemeinden. Egov Rev 11:18-19

Hilgers D (2012) Open government. Z Betriebswirtsch 82(6):631-660

Johnson RB, Onwuegbuzie AJ (2004) Mixed methods research: a research paradigm whose time has come. Educ Res 33(7):14-26

Pautz H (2009) Germany's social democrats in search of a new party programme. Politics 29(2):121-129

Piller F, Reichwald R (2009) Interaktive Wertschöpfung und Open Innovation. In: Picot A, Doeblin S (Hrsg) Innovationsführerschaft durch Open Innovation. Springer, Berlin, Heidelberg

Rapp M, Hoffmann C, Kröger N (2015) Beteiligung an open government fördern. HMD 52(1):161-171

Rapp M, White K, Rhomberg M (2016) Integrating the crowd through social media: how higher education can profit from viral mechanisms. In: Social computing and social media. Lecture notes in computer science 9742, S 394-404

Schmidthuber L, Hilgers D (2018) Unleashing innovation beyond organizational boundaries: exploring citizensourcing projects. Int J Public Adm 41(4):268-283

Schmidthuber L, Hilgers D, Gegenhuber T, Etzelstorfer S (2017) The emergence of local open government: determinants of citizen participation in online service reporting. J Gov Inf 34(3):457-469

Thomke SH (2003) Experimentation matters: unlocking the potential of new technologies for innovation. Harvard Business Press, Boston 